\title{
Anti-proliferative and Apoptotic Effects of Basella rubra (L.) Against 1, 2-Dimethyl Hydrazine-induced Colon Carcinogenesis in Rats
}

\author{
Bhanu Priya Kilari ${ }^{1}$, Venkata Subbaiah Kotakadi ${ }^{2 *}$, Josthna Penchalaneni ${ }^{1 *}$
}

\begin{abstract}
Colorectal cancer is a very prevalent diagnosed cancer. The current study was performed in order to examine the role of BRAE (Basella rubra aqueous extract) in regulating aberrant crypt foci (ACF) formation, cell proliferation and inhibition of apoptosis in a colon carcinogenesis model in male Wistar rats. Rats were randomly allocated into six groups. Group I served as control, and group II acted as a drug control administered BRAE $(250 \mathrm{mg} / \mathrm{kg} \mathrm{b.w.)}$ orally for 30 weeks. Rats in group III-VI were given subcutaneous injections of DMH $(25 \mathrm{mg} / \mathrm{kg}$ b.w. weekly) for 15 weeks to initiate colon carcinogenesis. Those in group IV and VI were administered BRAE along with DMH injections. Rats in group $V$ were administered with BRAE after cessation of DMH injection. After 30 weeks of experimental period colons were obtained from experimental groups and analyzed for ACF incidence, argyrophilic nucleolar organizing region- associated proteins (AgNOR) count, histopathological and immunohistochemical changes. Only in DMH exposed groups were ACF and AgNOR numbers increased. Administration of BRAE appreciably decreased the numbers of ACF and AgNOR in BRAE treated groups. Histopathological findings revealed a high level of dysplastic changes with decreased number of goblet cells found only in only DMH injected rats. Administration of BRAE in treated group rats reversed these changes. Expression markers for cell proliferation (PCNA and Ki67) were elevated in DMH treated rats, but reduced with BRAE treatement. This expression was reversed with apoptosis markers (p53 and Caspase-3). Thus the results results of the present study were found to be significant and confirmed the potential efficacy of BRAE against colon carcinogenesis.
\end{abstract}

Keywords: Basella rubra- 1, 2-dimethylhydrazine (DMH) - colon cancer- expression markers

Asian Pac J Cancer Prev, 17 (1), 73-80

\section{Introduction}

Colorectal cancer is the third most diagnosed cancer and it is a prime cause of morbidity and mortality in worldwide (Gellad et al., 2010; American Cancer Society, 2014). The occurrence of colorectal cancer is alarmingly increasing in Asian countries (Kirati et al., 2015). According to the epidemiological survey 12 million cancer cases were diagnosed so far, among those 7 million deaths occurred worldwide and these would be doubled by the end of the year 2030 (Burali Sanganna et al., 2013). The etiological factors for colon cancer development include both environmental and genetic factors (Juozas et al., 2014).

Humans exposed to hydrazine derivatives through environmental pollution and food contamination in day to day life (Ratasark et al., 2014). 1, 2-dimethylhydrazine $(\mathrm{DMH})$ is an environment pollutant and eminent experimental model to study the pathogenesis of colon cancer in rodents. This animal model resembles the way of human exposure to environment carcinogen. Dimethylhydrazine is a cancer causing substance that has ability to induce the colon cancer. It is a procarcinogen, metabolically activated in liver by a step wise reactions through intermediates like azoxymethane (AOM) and methylazoxy methane (MAM) and finally into carcinogenic metabolite methyldiazonium ion. This active carcinogen is transported to the colon via bile and blood. The formation of methyldiazonium ion in the body of rats induces DNA damage and mutations (Perse et al., 2011; Hamiza et al., 2012).

The morphological changes observed in rodents by 1,2-dimethylhydrazine induced colon carcinogenesis are the aberrant crypt foci (ACF). This is the first symptom of colon carcinogenesis. It is a preneoplastic lesion appears in rodent colons in early period after the carcinogen exposure of two weeks (McLellan et al., 1991). It increases in size and number, display the features of dysplasia 
(Bird 1987; Tudek 1989). The ACF have propensity to extend into adenomas and adenocarcinomas (Alrawi et al., 2006). During the progression of DMH-induced colon carcinogenesis in rats, it shows the related pathological features of human colon cancer (Venkatachalam et al., 2013).

The epidemiological studies suggest that life style and dietary habits plays prime role in activation or suppression of colon cancer development (Lofano et al., 2012). Adaptation of diet rich in fruits and vegetables are believed to be protective against colon cancer progression. A variety of compounds present in fruits and vegetables possess great anti-oxidant and anti-inflammatory properties (Das et al., 2007). Prevention of cancer through diet-based interference is considered as safe without any side effects (Venkatachalam et al., 2013).

The current study intended to determine the preventive or protective effects of Basella rubra. B.rubra is a green leafy vegetable belongs to the family Basellaceae, which is a red-leaved variety, highly nutritious with good source of vitamins and minerals (Murakami et al., 2001; Toshiyuki et al., 2001; Lakshmi Prasuna et al., 2009). It is an edible plant and widely used as a green vegetable throughout the country and also worldwide (Dong et al., 2012). Basella rubra is an abundantly available leafy vegetable in almost all places of Asian countries. It is used in traditional medicine for various purposes (Nagarajan et al., 2012; Priya et al., 2015). A limited number of studies have been done on this plant regarding the anticancer activity, so the proposed work designed to explore its benefits against colon cancer. The present study was designed to investigate the preventive effects of Basella rubra aqueous leaves extract against DMH induced colon carcinogenesis in rats.

\section{Materials and Methods}

\section{Chemicals}

1, 2-dimethylhydrazine (DMH) and Methylene blue, Hematoxylin and eosin and Silver nitrate were purchased from Sigma-Aldrich, USA. Commercial kits for immunostaining of PCNA, Ki67 and p53 for detection of cell proliferation and apoptosis were procured from BioGenes, USA. Caspase-3 (1: 100 dilution) antibody was acquired from Themoscientific, USA. All other chemicals used for this study were of analytical grade and obtained from Hi-Media Laboratories, Mumbai, India.

\section{Animal maintenance}

Animal studies were performed as per institute animal ethics committee regulations and approved by the committee (Reg. No. 1077/PO/a/12/CPCSEA/SPMVVIEC/2014/02). Male Wistar rats with 120-150 g weights were used for this study. Rats were placed in plastic cages covered with wire mesh. Temperature was maintained at $22+2^{\circ} \mathrm{C}$ and a humidity of $55+5 \%$ under a $12 \mathrm{~h}$ light-dark cycle.

\section{Collection and Preparation of leaves extract}

Basella rubra leaves were collected from the surrounding areas of Tirupati, Andhra Pradesh during the month of October 2012 and the plant was identified and authenticated (Herbarium No. SPMVV/BT/BR1/2012) by Dr.N.Nagaraju, Head, Department of Botany, S.V.Arts College, Tirupati, Andhra Pradesh.

The leaves were chopped into pieces and subjected to shade dry. The dried material was made it to powder with blender. $25 \mathrm{~g}$ of powder was weighted out and mixed with $1000 \mathrm{ml}$ distilled water then the contents were mixed well and keep it for $24 \mathrm{~h}$. The supernatant was collected and it was subjected to extraction procedure. The extracted mixture was filtered and it was freeze- dried. The frozen material was weighted and re-dissolved with distilled water prior to use.

\section{Carcinogen treatments}

The 1, 2-dimethylhydrazine (DMH) (Sigma-aldrich) was dissolved in 1mM EDTA (freshly prepared before use) and the $\mathrm{pH}$ was adjusted to 6.5 with $1 \mathrm{mM}$ sodium carbonate. The rate were given a subcutaneous injection of DMH at a dosage of $25 \mathrm{mg} / \mathrm{kg}$ b.w. (once a week) for 15 weeks.

\section{Experimental design}

All these rats were randomly allocated into six groups. In each group 12 rats, 6 rats were used for ACF analysis and 6 rats for histopathology, AgNOR and Immunohistochemical studies.

Group I- Control (received normal saline during the experimental period).

Group II- Drug control (rats received the BRAE ( $250 \mathrm{mg} / \mathrm{kg} /$ day b.w. every day) orally for 30 weeks).

Group III- Carcinogen control (rats injected with DMH ( $25 \mathrm{mg} / \mathrm{kg}$ b.w.) once a week (s.c.) for first 15 weeks).

Group IV - Pre- initiation (rats received DMH (25mg/ $\mathrm{kg}$ b.w. weekly once for 15 weeks s.c.) and meanwhile BRAE administered orally ( $250 \mathrm{mg} / \mathrm{kg} /$ day b.w. every day) starting one week before the experiment and continued up to end of carcinogen injection (0-15 weeks).

Group V - Post-initiation (rats received DMH ( $25 \mathrm{mg} / \mathrm{kg}$ b.w. weekly once for 15 weeks s.c.) and also administration of BRAE orally $(250 \mathrm{mg} / \mathrm{kg} /$ day b.w. every day) started after the DMH termination and proceeds until the end of experiment (16-30 weeks).

Group VI - Entire period (rats received DMH (25mg/ $\mathrm{kg}$ b.w. weekly once for 15 weeks s.c.) and also BRAE administration orally from day one of the experiment (1-30 weeks) and continued for entire period of the experiment (0-30 weeks).

\section{Colon processing \& ACF quantification}

After necropsy organs and colons were collected from all the rats and they were observed for morphological changes. Organs were weighted and they were preserved in buffered formalin for further study. Colons were collected and they were cut opened longitudinally and rinse with $0.9 \%$ saline. Colons were flattened and fixed in $10 \%$ formalin. Then the colons were stained with $0.2 \%$ methylene blue dissolved in phosphate buffered saline (PBS) for analysis of ACF. Then the colon mucosal surface was examined for the number of ACF and counting done under a light microscope. 


\section{Histological examination}

Colons collected from the rats were Swiss-rolled and fixed in formalin (de Moura et al., 2012). Histopathological evaluation was carried out by hematoxylin-eosin (H\&E) staining to determine the histopathological changes in the colon sections of different experimental group rats.

\section{Cell proliferation assay by AgNOR Staining}

The colon sections were deparaffinized in xylene and hydrated through decreasing grades of ethanol to double distilled deionized water. Sections were then reacted with freshly prepared silver nitrate colloidal solution (1 part by $2 \%$ gelatin in $1 \%$ formic acid and 2 parts by volume of $50 \%$ aqueous silver nitrate solution) for 30 minutes at room temperature. (Assay was performed in dark environment). The silver colloidal solution was washed with double distilled ionized water. Then the sections were treated with 5\% sodium thiosulphate for 5 minutes and again washed with double distilled water. The sections were dehydrated through increasing grades of alcohol. At last the slides were cleaned in xylene and mounted using DPx.

AgNOR count were evaluated by cell nuclei of 25-well oriented crypts could be observed. Counting performed under a light microscope.

\section{Immunohistochemistry}

Immunohistochemistry staining of PCNA, Ki67 and p53 was performed as per instructions of kit (BioGenes, USA). Caspase- 3 expression was observed in the colon sections as per procedure of Khan et al., 2012. Primary antibody used was rabbit polyclonal anti-caspase-3 (PA516335, 1:100 dilution, Thermoscientific, USA).

\section{Statistical analysis}

The values obtained from results were articulated as the mean + standard deviation (SD). The statistical significance of the data was evaluated by One-way analysis of variance (ANOVA) followed by Dunnett's Comparision Test. The statistical significance was considered at $* \mathrm{p}<0.05$.

\section{Results}

Evaluation of BRAE efficacy on tumor frequency and number

The effect of BRAE on tumor frequency and number were shown in Table 1. In control (group I) and drug control rats (group II) no tumors were observed. Tumors were totally absent in those groups. Rats injected with only DMH (group III) a total of 6 rats display tumors in their colons. Totally 21 tumors were examined with $100 \%$ incidence. In group IV administration of BRAE to DMH induced rats, 5 rats display 15 tumors with $72 \%$ incidence. Administration of BRAE to DMH injected rats (group V), 4 rats display 10 tumors with $47 \%$ frequency. Administration of BRAE to DMH induced rats (group VI), 3 rats bear a total of 5 tumors with $24 \%$ incidence. Along with tumor number, size of the tumor were also

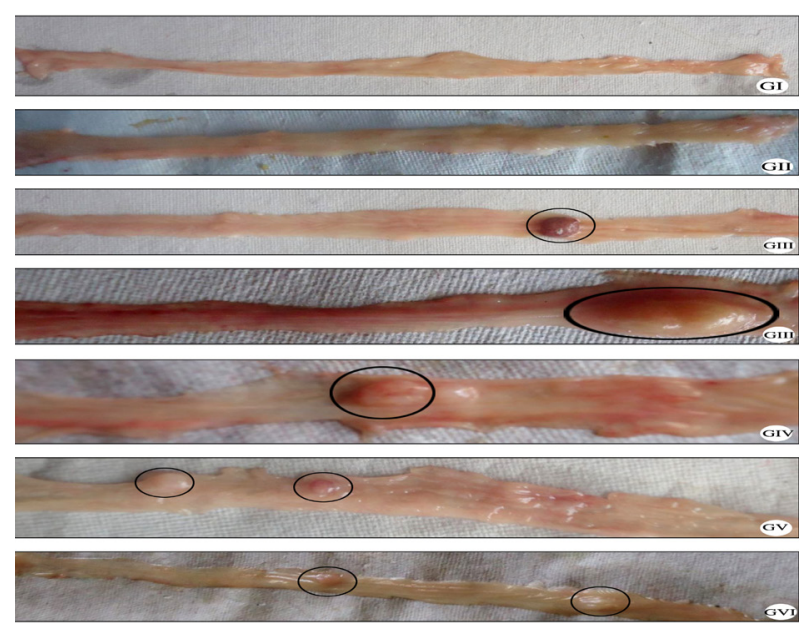

Figure 1. Morphological Observations in the Longitudinally Opened Colon Structures. (GI) Figure represents control group colon, (GII) Drug control colon, where GI \& GII shows absence of tumors in the colons; (GIII) Only DMH induced colons with large size tumors, (GIV) Colon from initiation group, $(\mathrm{GV})$ Colon in post-initiation group shows reduced number of tumors, (GVI) Colon in entire period group have tumors with reduced number and size

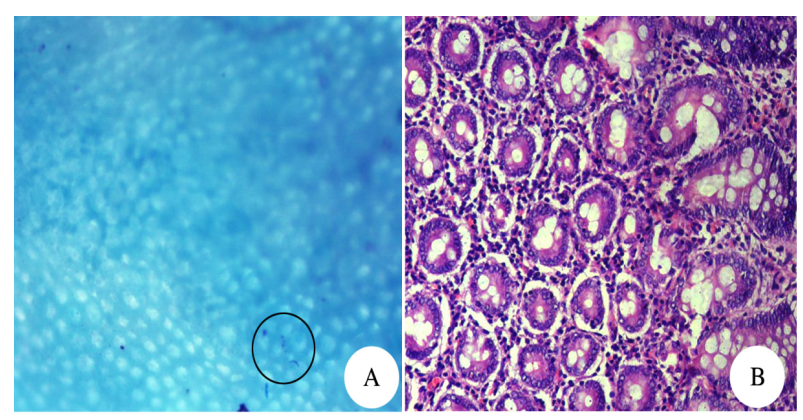

Figure 2.A) Methylene Blue Staining of Colon Section Represents ACF (10X objective). B) View of ACF in H\&E Section Showing Dysplasia. Aberrant crypt foci $(\mathrm{ACF})$ were produced by the injection of dimethylhydrazine

Table 1. Evaluation of BRAE Efficacy on Tumor Formation

\begin{tabular}{|c|c|c|c|c|}
\hline Groups & $\begin{array}{l}\text { Number } \\
\text { of rats }\end{array}$ & $\begin{array}{l}\text { Number of rats } \\
\text { with tumors }\end{array}$ & $\begin{array}{c}\text { Tumor } \\
\text { incidence }(\%)\end{array}$ & $\begin{array}{c}\text { Total number } \\
\text { of tumors }\end{array}$ \\
\hline Group I (Control) & 6 & NIL & NIL & NIL \\
\hline Group II (Drug Control) & 6 & NIL & NIL & NIL \\
\hline Group III (Only DMH) & 6 & 6 & 100 & 21 \\
\hline Group IV (Initiation) & 6 & 5 & 72 & 15 \\
\hline Group V (Post-initiation) & 6 & 4 & 47 & 10 \\
\hline Group VI (Entire period) & 6 & 3 & 24 & 5 \\
\hline
\end{tabular}

NIL - represents absence of tumor. In control and drug control tumor formation was not seen 
Table 2. Evaluation of BRAE Efficacy on ACF Number

\begin{tabular}{lccc}
\hline Groups & $\begin{array}{c}\text { Number ACF incidence Total number } \\
\text { of rats }\end{array}$ & $(\%)$ & of ACF/Colon \\
\hline Group I (Control) & 6 & NIL & NIL \\
Group II (Drug Control) & 6 & NIL & NIL \\
Group III (Only DMH) & 6 & 100 & $101.6 \pm 9.6^{\mathrm{a}}$ \\
Group IV (Initiation) & 6 & 100 & $62.2 \pm 8.6^{\mathrm{b}}$ \\
Group V (Post-initiation) & 6 & 100 & $45.8 \pm 10.6^{\mathrm{c}}$ \\
Group VI (Entire period) & 6 & 100 & $28.6 \pm 11.7^{\mathrm{d}}$ \\
\hline
\end{tabular}

Values are mean \pm S.D. Significant Vs group III. Values representing with (a-d) superscripts differ significantly at $\mathrm{p}<0.01$; NIL - represents absence of tumor. In control and drug control tumor formation was not seen

Table 3. Evaluation of BRAE Efficacy on AgNOR Number

\begin{tabular}{lccc}
\hline Groups & $\begin{array}{c}\text { Number } \\
\text { of rats }\end{array}$ & $\begin{array}{c}\text { Number } \\
\text { of crypts } \\
\text { observed }\end{array}$ & $\begin{array}{c}\text { Number } \\
\text { of AgNORs/ * } \\
\text { nucleus }\end{array}$ \\
\hline Group I (Control) & 6 & 25 & $1.80 \pm 0.28 \mathrm{a}$ \\
Group II (Drug Control) & 6 & 25 & $1.92 \pm 0.32^{\mathrm{a}}$ \\
Group III (Only DMH) & 6 & 25 & $3.38 \pm 0.48^{\mathrm{b}}$ \\
Group IV (Initiation) & 6 & 25 & $2.8 \pm 0.32^{\mathrm{c}}$ \\
Group V (Post-initiation) & 6 & 25 & $2.57 \pm 0.33^{\mathrm{d}}$ \\
Group VI (Entire period) & 6 & 25 & $2.2 \pm 0.20^{\mathrm{e}}$ \\
\hline
\end{tabular}

Experimental values are expressed as mean $\pm \mathrm{SD}$; Values representing with (a-e) superscripts differ significantly at $\mathrm{p}<0.05$; *AgNORs/nucleus values in each group represent the level of proliferation
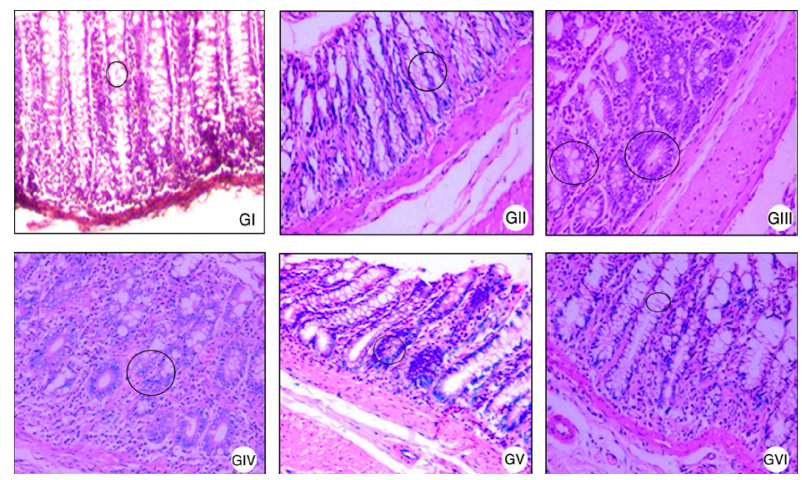

Figure 3. Topographical View of Histological Alterations in the Colon Sections (20X Objective). (GI) Represents control group sections of normal appearance of glandular structure. (GII) Drug control colon sections with normal appearance of intestinal lining epithelial cells with goblet cells. (GIII) Only DMH induced sections showing proliferation of intestinal lining epithelial cells with dysplastic changes. (GIV) Sections in initiation group showing hyperplasia with dysplastic changes. (GV) Sections in post-initiation group showing crypt epithelial cells with mild hyperplasic changes. (GVI) Sections from entire period group showing normal appearance of crypt cells with increased number of goblet cells.

reduced in post-initiation (Group V) and entire period (Group VI) treatments with BRAE compared to only DMH induced (group III) rats. Tumor occurrences in different experimental group rats were displayed in Figure1.

\section{Evaluation of BRAE efficacy on ACF formation}

In control (group I) and drug control group (group
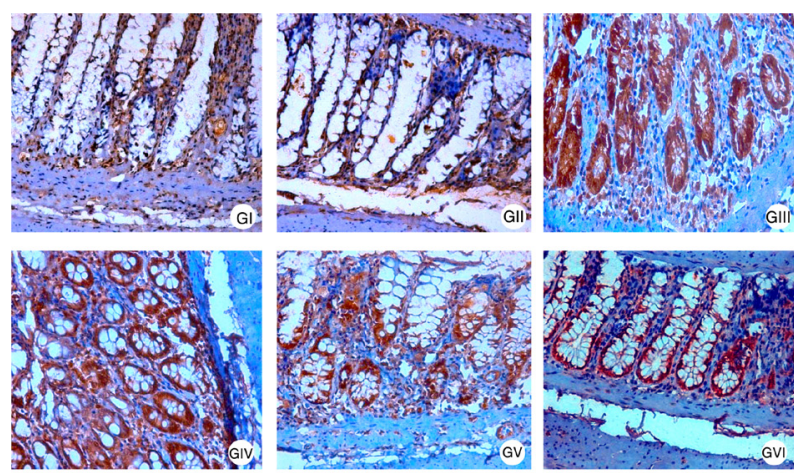

Figure 4. Immunohistochemical Staining for PCNA (20X Objective). Brown colour represents PCNA specific staining, blue colour represents haemotoxylin staining. (GI) and (GII) are control and drug control colon sections with minimal immunoreactivity towards PCNA. (GIII) Only DMH induced sections explore more intense immunopositive staining compared to control section. (GIV) In initiation group sections, PCNA expression was reduced compared to group III. (GV) BRAE adminstration in post-initiation shows lesser PCNA expression. (GVI) Entire period adminstration of BRAE shows potent inhibition of PCNA
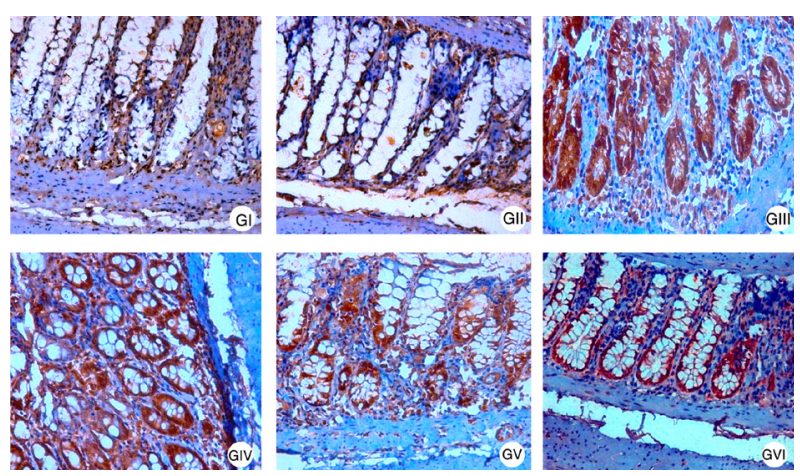

Figure 5. Immunohistochemical Staining for Ki67 (20X Objective). Brown colour represents Ki67 specific staining, blue colour represents haemotoxylin staining. (GI) and (GII) are control and drug control colon sections with minute indication for Ki67. (GIII) Only DMH induced sections possess significantly increased expression of Ki67 compared to control section. (GIV) In initiation group sections, Ki67 indication was reduced to some extent. (GV) BRAE adminstration in post-initiation and entire period treatments shows lesser Ki67 indication. (GVI) Appreciable results were observed in entire period adminstration of BRAE

II) no ACF were observed in the rat colons. The total number of ACF was higher in only DMH induced rats (group III). These results displayed that the rats induced with DMH produces ACF in colons. Administration of BRAE to DMH induced rats reduces the ACF number in initiation (group IV), post-initiation (group V) and entire period (group VI) treatments. Reduction was more considerably significant $(\mathrm{p}<0.01)$ in entire period group rat colons compared to group III. Inhibitions of ACF in BRAE administration groups were depicted in Table 2 and Figure 2.

Evaluation of BRAE efficacy on histopathological changes Histopathological alterations in the colon sections of 

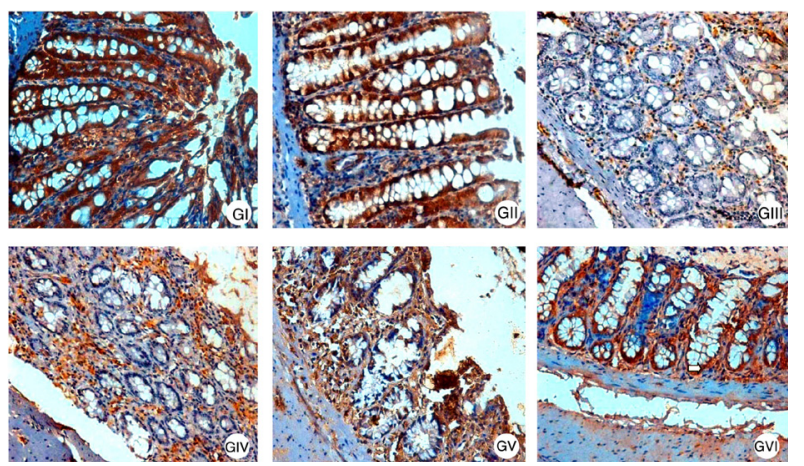

Figure 6. Immunohistochemical Staining for p53 (20X

Objective). Brown colour represents p53 specific staining, blue colour represents haemotoxylin staining. (GI) and (GII) are control and drug control colon sections with greater expression towards p53. (GIII) Only DMH induced sections shows strong reduction of p53 expression compared to control section. (GIV) In initiation group sections p53 expression was increased compared to group III. (GV) BRAE adminstration in postinitiation shows increased expression of p53.(GVI) Outstanding results were shown in entire period sections
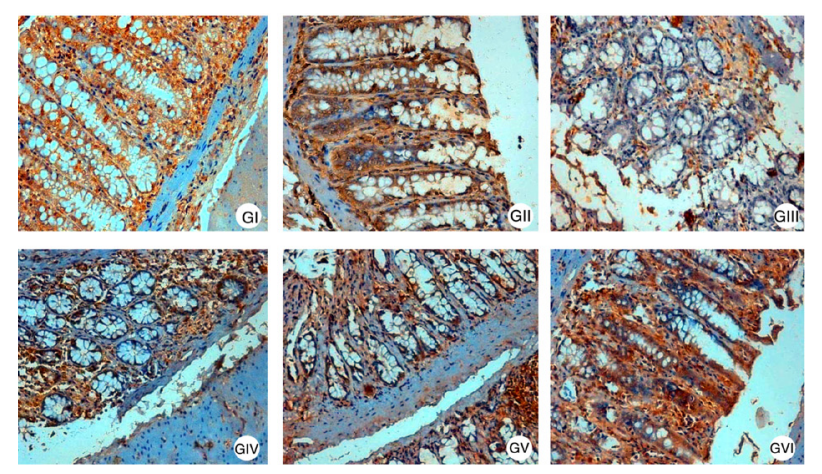

Figure 7. Immunohistochemical Staining for Caspase-3 (20X Objective). Brown colour symbolize caspase-3 staining, blue colour symbolize haemotoxylin staining. (GI) and (GII) are control and drug control colon sections shows immmunopositive staining of caspase-3. (GIII) Only DMH induced rats have immunonegative staining towards caspase- 3 compared to control section. (GIV \& GV) BRAE adminstration in initiation and post-initiation shows expression of caspase-3. (GVI) More pronounced expression was observed in entire period sections

rats were represented in Figure 3. Control rats (group I) had normal appearance of intestinal glandular structure. Rats administered with only BRAE (group II) had normal appearance of intestinal lining epithelium cells with no abnormalities. Only DMH injected rats (group III) shows hyper proliferation of crypt epithelial cells with anaplastic changes, cell proliferation of tumor cells in sub mucosa indicating invasive changes. Administration of BRAE to DMH induced rats (group IV \& V) had normal appearance of crypt epithelium cells with goblet cells and mild hyperplasia. Colon sections in group VI rats restore the changes to normal appearance and more pronounced outcome was seen in this group with increased goblets cells.
Evaluation of BRAE efficacy on the AgNORs number/ nucleus

Number of AgNORs in the crypts of all group rats was displayed in Table 3. Only DMH injected rats (group III) shows more AgNOR/ nucleus count compared to all other groups. It is evident that $\mathrm{DMH}$ induced the cell hyper proliferation that leads to cancer initiation. The AgNOR/ nucleus was less in BRAE administration groups (IV, $\mathrm{V}$ $\& \mathrm{VI})$. BRAE shows its potential activity in entire period regime (group VI) by significantly $(\mathrm{p}<0.05)$ decreasing the AgNOR count and cell proliferation compared to group III.

\section{Evaluation of BRAE efficacy on cell proliferation and apoptosis}

The expression of cell proliferation markers (PCNA \& Ki67) were depicted in Figure 4 and 5. Expression of apoptosis markers (p53 \& Caspase-3) were depicted in Figure 6 and 7. There was no divergence in the immunostaining of four proteins in group II as compared to group I. The sections of only DMH injected rats (group III) have high levels of PCNA \& Ki67 expression and less expression towards 553 \& Caspase-3.

Administration of BRAE to DMH induced rats (group IV, $\mathrm{V} \& \mathrm{VI}$ ) reduces the expression of cell proliferation markers (PCNA \& Ki67) and elevates the expression of apoptotic markers (p53 \& Caspase-3). In the entire period administration of BRAE potently inhibited the PCNA \& Ki67 indication and profoundly stimulated p53 $\&$ Caspase- 3 expression. The results provided evidence that the BRAE might activate the apoptosis by inhibiting the cell proliferation in cancer cells.

\section{Discussion}

The experimental studies revealed that the most of the cases of colorectal cancer are environmental origin so it can be preventable (Lund et al., 2006). The epidemiological reviews also declared that the consumption of diet rich with antioxidant nutrients may reduces the risk of oxidative stress- related disorders (Guizani et al., 2013). Several reports explored that naturally occurring phenolics in fruits and vegetables as a combination can prevent cancer cell growth more efficiently than as a single compound (Halliwell et al., 2007). Numerous studies reported that higher intake of fruits and vegetables have a protective role against colorectal cancer (Van et al., 2009). Everyone knows the importance of green leafy vegetables but their consumption was highly condensed and ignored. This study aims to highlight the significance of intake of leafy vegetable (Basella rubra) in our regular diet.

Basella rubra aerial parts are used as leafy vegetable and healthy staple food (Murakami et al., 2001). Leaves of $\mathrm{B}$. rubra contains essentials nutrients like proteins, fat, vitamin $C$, vitamin $A$, vitamin $E$, vitamin $K$, vitamin B9 (folic acid), riboflavin, thiamine, niacin and minerals like calcium, magnesium and iron (Adhikari et al., 2012). Many compelling findings also suggest that the deficiency of folate increases the chance of risk of colorectal cancer (Kim 1999). Keeping in view the above fact we have selected the plant B .rubra which possess enough or high 
amount of folic acid. It is apparent that diet loaded in vegetables prevents the consumers from gastrointestinal problems, so it is more encouraged to eat diet rich with vegetables (Katarzyna et al., 2007). The present experimental study was conducted in three different stages (initiation, post-initiation and entire period) in order to determine on which stage administration of B.rubra will work out effectively against colon cancer. So the present study performed to evaluate the chemopreventive efficacy of B.rubra against colon carcinogenesis in rats by long term bioassay.

The DMH (1, 2-dimethyl hydrazine) usage has overcome the use of other chemical carcinogens. DMH is the most reproducible model for cancer researchers to study the disease progression (Rosenberg et al., 1995). DMH used in the present study is thus justifiable model for inducing colon cancer in male Wistar rats.

ACF were distinguished from normal crypts by their elliptical shape, large size and darker staining (Rodrigues et al., 2002). Administration of BRAE suppresses the number of ACF and also the progression of preneoplastic to malignant neoplasia. BRAE inhibitory effects were explored through different phases of carcinogenesis (initiation, post-initiation and entire period). Total number of ACF was elevated in only DMH induced rats (group III). Significant reductions of total number of ACF were seen in colons of entire period administration of BRAE rats (group VI). The results showed that BRAE plays an important role in inhibiting formation of ACF and constant and rapid reduction of ACF in BRAE treated groups.

The loss of differentiation of goblet cells is regarded as a prime feature of malignant preneoplastic lesions (Tanaka et al., 2009). In our histology reports, rats injected with only DMH (group III) revealed sections of crypt epithelial cells proliferation with more anaplastic, dysplastic, pleomorphic and hyperchromatid changes with loss of goblet cells. Abdelkader et al., 2012 states that goblet cells are intestinal secretory cell lineages that results the inhibition of colon carcinogenesis by the mucin release (Muc2). Our findings agreed with above statement. Whereas the colon sections in BRAE administration to DMH induced rats (group IV, V \& VI) have normal appearance of crypt epithelial cells with goblet formation and colonic tumors multiplicity was greatly reduced in BRAE treatment groups.

Nucleolar organizer regions (NOR) are loops of DNA that possess ribosomal RNA genes. NOR-related acidic proteins are interrelated to sites of rRNA transcription that can be established in the interphase nucleus by silver-staining. In this method, the products are appeared as black dots, known as AgNORs. Abnormal cell proliferation in colonic epithelium is regarded to be high risk of colon cancer. Ameilinda et al., 2011 reported that cancer cells have increased or more number of AgNORs than the normal cells. The number of AgNOR dots in nucleus is used to calculate the cell proliferation index (Sengottuvelan et al., 2006). Our results showed that the rats injected with only DMH demonstrates increased AgNORs count, where as rats administered with BRAE shows decreased number of AgNORs resemble decreased cell proliferation in three stages (group IV, V \& VI).
Among three stages, administration of BRAE in entire period (group VI) offers effective reduction of AgNORs dots/nucleus.

Proliferative cell nuclear antigen (PCNA) is non-histone nuclear acidic protein indication in the proliferating cells nuclei in G1 and S phase of the cell cycle (Hamiza et al., 2012). PCNA is known to be an important gastrointestinal biomarker (Nagendraprabhu et al., 2011). Expression of PCNA indicates the cell proliferation activity and it is used as an index in evaluating tumor cell proliferation.

Apoptosis plays a down-regulative role in the development of tumors (Xu et al., 2010). In the current study, PCNA expression was high in DMH induced rats (group III) where this was reverse in administration of BRAE for entire period rats (group VI). BRAE profoundly inhibits the PCNA expression in entire period group.

The nuclear antigen, Ki67 is also a cell proliferation marker. It is particularly expressed in $\mathrm{G} 1, \mathrm{~S}$, and $\mathrm{G} 2$ phases of cell cycle but not in G0 phase (Katsuki et al., 2006). Our results showed that Ki67 expression was highly increased in only DMH induced rats (group III) while administration of BRAE significantly decreased the expression of Ki67 in rat colons. Effective reduction was found in group VI rats compared to group $\mathrm{V}$ and group IV rats.

The p53 is a tumor suppressor protein. It regulates the transcription of genes involved in cell cycle, DNA repair and apoptosis. Apoptosis is a method through which damaged cells are removed during colonic crypt cell homeostasis. This process can also be evaluated by determination of caspase-3 activity. Caspases are crucial mediators in apoptotic pathway (Porter et al., 1999).

Interestingly, in our results we observed the reduced positive staining of p53 towards only DMH induced rats (group III). Increased immunopositive staining of p53 was observed in BRAE administration to DMH injected rats (group VI). The same type of results was seen for caspase-3. DMH injected (group III) rats showed less immunopositive staining towards caspase-3. Administration of BRAE to entire period (group VI) rats shows enhanced immunopositive staining of caspase-3. Administration of BRAE for entire period exerts admirable effects on inhibition of proliferation and promotion of apoptosis in colon cancer cells. Similar type of immunohistochemical (p53 marker) result was reported by previous reports using plant extracts like Ginko biloba and Ginseng (Kotakadi et al., 2008; Jin et al., 2008).

Based on the results from the current study, the ACF enumeration, histological evaluation, cell proliferation count and the expression of apoptosis markers proposed that aqueous extract of Basella rubra as a potent chemotherapeutic agent. B. rubra is effective in entire period treatment towards the inhibition of cell proliferation by activating the apoptosis mechanism in chemically induced colon carcinogenesis. This action may be due to the property of inducing caspase-dependent apoptosis in cancer cells. Cumulatively, the study suggests that consumption of the Basella rubra in regular diet has protective and preventable effects towards colon cancer. The present study highly specifies that B. rubra own anticancer activity and therefore it can be use as effectual therapeutic agent for treatment of colon cancer. 


\section{Acknowledgements}

The authors highly thankful to Prof. CH. Srilatha, Head, Department of Pathology, Sri Venkateswara Veterinary University, Tirupati, A.P. for assisting and guiding in histology and immunohistology slides and also grateful to DST-PURSE, Sri Venkateswara University, Tirupati, A.P. for providing instrumentation facilities.

\section{References}

Abdelkader B, Djerdjouri B, Laroche-Clary A, et al (2012). Short curcumin treatment modulates oxidative stress, arginase activity, aberrant crypt foci, and TGF-1 and HES-1 transcripts in 1,2-dimethylhydrazine-colon carcinogenesis in mice. Toxicol, 302, 308-17.

Adhikari R, Naveen Kumar HN, Shruthi SD (2012). A review on medicinal importance of Basella alba L. Int J Pharm Sci Drug Res, 4, 110-14.

Alrawi SJ, Schiff M, Carroll RE, et al (2006). Aberrant crypt foci. Anticancer Res, 26,107-9.

Ameilinda M, Farida S, Putri LW, et al (2011). Antiproliferative activity of ethanolic extract of Ciplukan herbs (Physalis angulata L.) on 7, 12-Dimethylbenz[a]nthracene-induced rat mammary carcinogenesis. Indonesian J Cancer Chemoprevent, 2, 227-32.

Bird RP (1987). Observation and quantification of aberrant crypts in the murine colon treated with a colon carcinogen: Preliminary findings. Cancer Lett, 2, 147-51.

Colorectal Cancer Facts \& Figures 2014-2016 (2014). http:// www.cancer.org/acs/groups/content/ @ epidemiology. Surveillance/documents/document/acspc-042280.pdf.

Das D, Arber N, Jankowski JA (2007). Chemoprevention of colorectal cancer. Digest, 76, 51-67.

de Moura NA, Caetano BFR, Sivieri K, et al (2012). Protective effects of yacon (Smallanthus sonchifolius) intake on experimental colon carcinogenesis. Food Chem Toxicol, 50, 2902-10.

Dong C-A, Hayashi K, Mizukoshi Y, et al (2012). Structures and anti-HSV-2 activities of neutral polysaccharides from an edible plant, Basella rubra L. Intl J Biol Macromol, 50, 245-9.

Guizani N, Ibrahim Waly M, Rahman MS (2013). Nabag (Zizyphus spina-christi) extract prevents aberrant crypt foci development in colons of azoxymethane-treated rats by abrogating oxidative stress and inducing apoptosis. Asian Pac J Cancer Prev, 14, 5031-35.

Gellad ZF, Provenzale D (2010). Colorectal cancer: National and international perspective on the burden of disease and public health impact. Gastroenterol, 138, 2177-90.

Haenszel W, Correa P (1973). Cancer of the large intestine. Epidemiological findings. Dis Colon Rectum, 16, 371-77.

Halliwell B (2007). Dietary polyphenols: good, bad, or indifferent for your health? Cardiovasc Res, 73, 341-7.

Hamiza OO, Muneeb U Rehman, Mir Tahir, et al (2012). Amelioration of 1, 2-dimethylhydrazine (DMH) induced colon oxidative stress, inflammation and tumor promotion response by tannic acid in Wistar rats. Asian Pac J Cancer Prev, 13, 4393-402.

Jin Y, Kotakadi VS, Ying L, et al (2008). American ginseng suppresses inflammation and DNA damage associated with mouse colitis. Carcinogenesis, 29, 2351-9.

Katarzyna P, Bennett RN, Kromer K, et al (2007). Assessment of the antiproliferative activity of carrot and apple extracts, Pol J Food Nutr Sci, 57, 307-14.

Katsuki T, Hirata K, Ishikawa H, et al (2006). Aged garlic extract has chemopreventative effects on 1,2-dimethylhydrazineinduced colon tumors in rats. J Nutr, 136, 847-51.

Khan R, Khan AQ, Qamar W, et al (2012). Chrysin protects against cisplatin-induced colon toxicity via amelioration of oxidative stress and apoptosis: probable role of p38 MAPK and p53. Toxicol Appl Pharmacol, 258, 315-29.

Kim Y-N (1999). Folate and carcinogenesis: Evidence, mechanisms, and implications. J Nutr Biochem, 10, 66-88.

Kotakadi VS, Jin Y, Hofseth AB, et al (2008). Ginkgo biloba extract EGb 761 has anti-inflammatory properties and ameliorates colitis in mice by driving effector $\mathrm{T}$ cell apoptosis. Carcinogenesis, 29, 1799-1806.

Kupcinskas J, Gyvyte U, Bruzaite I, et al (2014). Common genetic variants of PSCA, MUC1 and PLCE1 genes are not associated with colorectal cancer. Asian Pac J Cancer Prev, 16, 6027-32.

Lakshmi Prasuna CP, Chakradhar RPS, Rao JL, et al (2009). EPR and IR spectral investigations on some leafy vegetables of Indian origin. Spectrochim Acta Part A, 74, 140-7.

Lofano K, Principi M, Scavo MP, et al (2012). Dietary lifestyle and colorectal cancer onset, recurrence and survival: myth or reality? J Gastrointest Cancer, 44, 1-11.

Lund EK (2006). Dietary fatty acids and colon cancer. Scand $J$ Food Nutr, 50, 39-44.

McLellan EA, Medline A, Bird RP (1991). Sequential analyses of the growth and morphological characteristics of aberrant crypt foci: putative preneoplastic lesions. Cancer Res, $\mathbf{5 1}$,

Murakami T, Hirano K, Yoshikawa M (2001). Medicinal foodstuffs. XXIII. Structures of new oleanane-type triterpene oligoglycosides, basellasaponins A, B, C, and D, from the fresh aerial parts of Basella rubra L. Chem Pharm Bull, 49, 776-9.

Nagarajan A, Gomathi R, Manian S, et al (2012). Evaluation of Basella rubra 1., rumex nepalensis spreng. and Commelina benghalensis L. for antioxidant activity. Int J Pharm Pharm Sci, 4, 714-20.

Nagendraprabhu P, Sudhandiran G (2011). Astaxanthin inhibits tumor invasion by decreasing extracellular matrix production and induces apoptosis in experimental rat colon carcinogenesis by modulating the expressions of ERK-2, NF k-B and COX-2. Invest New Drugs, 29, 207-24.

Perse M, Cerar A (2011). Morphological and molecular alterations in 1,2 dimethylhydrazine and azoxymethane induced colon carcinogenesis in rats. J Biomed Biotechnol, 473964, 1-14.

Porter AG, Janicke RU (1999). Emerging roles of caspase-3 in apoptosis. Cell Death Differ, 2, 99-104.

Poomphakwaen K, Promthet S, Suwanrungruang K, et al (2015). Risk factors for colorectal cancer in Thailand. Asian Pac J Cancer Prev, 16, 6105-09.

Priya K, Ankur Gupta, Surabhi Mahajan, et al (2015). Evaluation of antimicrobial properties of Basella rubra methanolic extracts on selected microorganisms. Int J Pharm Sci Res, 6, 334-6.

Rodrigues MAM, Silva LAG, Salvadori DMF, et al (2002). Aberrant crypt foci and colon cancer: comparison between a short- and medium -term bioassay for colon carcinogenesis using dimethylhydrazine in Wistar rats. Braz J Med Bio Res, 35, 351-5.

Rosenberg DW, Liu Y (1995). Induction of aberrant crypts in murine colon with varying sensitivity to colon carcinogenesis. Cancer Lett, 92, 209-14.

Sanganna B, Kulkarni AR (2013). Effect of Citrus reticulata essential oil on aberrant crypt foci (acf) development in1, 2-dimetylhydrazine induced colon carcinogenesis rats. Int $J$ Pharmaceut Appl, 4, 29-37.

Sengottuvelan M, Nalini N (2006). Dietary supplementaᄀtion 
K Bhanu Priya et al

of resveratrol suppresses colonic tumour incidence in 1,2-dimethylhydrazine-treated rats by modulating biotransforming enzymes and aberrant crypt foci deᄀvelopment. Br J Nutr, 96, 145-53.

Summart R, Chewonarin T (2014). Purple rice extract supplemented diet reduces DMH-induced aberrant crypt foci in the rat colon by inhibition of bacterial $\beta$-glucoronidase. Asian Pac J Cancer Prev, 15, 749-55.

Tanaka T, Yasui Y, Tanaka M, et al (2009). Melatonin suppresses AOM/DSS-induced large bowel oncogenesis in rats. Chem Biol Interact, 177, 128-36.

Tudek B, Bird RP, Bruce WR (1989). Foci of aberrant crypts in the colons of mice and rats exposed to carcinogens associated with foods. Cancer Res, 49, 1236-40.

Van Duijnhoven FJ, Bueno-De-Mesquita HB, Ferrari P, et al (2009). Fruit, vegetables and colorectal cancer risk: the European Prospective Investigation into Cancer and Nutrition. Am J Clin Nutr, 89, 1441-52.

Venkatachalam K, Gunasekaran S, Jesudoss VA, et al (2013). The effect of rosamarinic acid on 1,2-dimethylhydrazine induced colon carcinogenesis. Exp Toxicol Pathol, 65, 409-18.

Xu G, Ren G, Xu X, et al (2010). Combination of curcumin and green tea catechins prevents dimethylhydrazine- induced colon carcinogenesis. Food Chem Toxicol, 48, 390-5. 